Article

\title{
Vibration and Flutter of a Honeycomb Sandwich Plate with Zero Poisson's Ratio
}

\author{
Junhua Zhang ${ }^{1, * \mathbb{D}}$, Zhaochen Yan $^{1}$ and Lili Xia ${ }^{2}$ \\ 1 College of Mechanical Engineering, Beijing Information Science and Technology University, Beijing 100192, \\ China; yzc_wr@163.com \\ 2 College of Sciences, Beijing Information Science and Technology University, Beijing 100192, China; \\ xialili@bistu.edu.cn \\ * Correspondence: zjhuar@163.com
}

check for updates

Citation: Zhang, J.; Yan, Z.; Xia, L. Vibration and Flutter of a Honeycomb Sandwich Plate with Zero Poisson's Ratio. Mathematics 2021, 9, 2528. https://doi.org/10.3390/ math9192528

Academic Editor: Hari Mohan Srivastava

Received: 29 August 2021

Accepted: 1 October 2021

Published: 8 October 2021

Publisher's Note: MDPI stays neutral with regard to jurisdictional claims in published maps and institutional affiliations.

Copyright: (c) 2021 by the authors. Licensee MDPI, Basel, Switzerland. This article is an open access article distributed under the terms and conditions of the Creative Commons Attribution (CC BY) license (https:/ / creativecommons.org/licenses/by/ $4.0 /)$.

\begin{abstract}
A honeycomb is a kind of excellent lightweight structure and a honeycomb sandwich plate with zero Poisson's ratio (ZPR) core is used widely in morphing structures. In this paper, a sandwich plate composed of a honeycomb core with zero Poisson's ratio is analyzed for free vibrations and flutter under supersonic airflows. The equivalent elastic parametric formulas of the honeycomb core for zero Poisson's ratio are proposed. The models are compared for their natural frequencies by theoretical and finite element methods respectively, which verifies the validity of the equivalent elastic parametric formulas and the model for the honeycomb sandwich plate with zero Poisson's ratio. The influence of the geometric parameters of the honeycomb plate on the vibration frequencies is obtained. Three kinds of honeycomb cores, namely, regular hexagon, auxetic and hybrid with zero Poisson's ratio, are compared through natural frequencies of the sandwich plate. It is found that the frequency of the zero Poisson's ratio honeycomb sandwich plate is the second one when the other parameters are the same. The flutter of the honeycomb plate is analyzed by using the first order piston theory under supersonic flows. The critical flutter velocity of the plate is obtained, and the influence of geometric parameters of the honeycomb plate on the critical flutter velocities is obtained.
\end{abstract}

Keywords: honeycomb sandwich plate; zero Poisson's ratio; vibration; flutter

\section{Introduction}

Honeycomb structures have received extensive applications in engineering and daily life, such as aerospace, building, vehicle, and packaging, etc., as they have outstanding mechanical properties such as lightweight, low weight-to-stiffness ratios, good energy absorption characteristics, etc. Moreover, the geometry of the unit cells of honeycombs can be tailored easily to result in desired deformed shapes under loading which allow for abnormal or expected behaviors in macroscale. Hence, increasing numbers of studies have focused on honeycomb structures. The energy absorption of the honeycomb sandwich structure was increased by adding an absorbent agent to the Nomex honeycomb wall that was filled with polymer tube [1]. Rapaka et al. [2] studied the dynamic compressive behaviors of hexagonal honeycombs under shocks. Rahman and Koohbor [3] studied the energy absorption performances of density gradient honeycombs. Zhang et al. [4,5] investigated the dynamic crushing responses of honeycombs with negative Poisson's ratio. Jin et al. [6] investigated the vibration suppression of the square honeycomb sandwich structure.

Honeycomb sandwich structures with zero Poisson's ratio have the outstanding advantages of that deformation in one direction that does not affect other directions. Therefore, they are widely used in flexible skin for morphing structures such as morphing aircraft and wings. The in-plane mechanical properties of the honeycomb layer is very important for analysis of honeycomb sandwich structures. Olympio and Gandhi [7] proposed a sandwich skin for morphing aircraft, comprised of cellular honeycomb core with zero Poisson's ratio and studied mechanical properties of the honeycomb. Chen 
et al. [8] investigated in-plane mechanical properties of zero-Poisson cell structures by using analytical, numerical and experimental methods simultaneously. Bubert et al. [9] gave the equivalent elastic parameters of an accordion-like flexible honeycomb used as morphing aircraft skin. Zou et al. [10] proposed a zero Poisson's ratio mixed cruciform honeycomb and studied the in-plane elastic constants, through theoretical calculation, numerical simulation and experiments. Liu et al. [11] proposed a trapezoidal honeycomb with close-to-zero Poisson's ratio and the effects of geometric parameters on the elastic properties of the structure were studied. Huang et al. [12,13] proposed a close-to-zero Poisson's ratio honeycomb with trapezoidal beams and investigated the multi-stiffness topology optimization of the zero Poisson's ratio honeycomb.

$\mathrm{Xu}$ et al. $[14,15]$ proposed hybrid AuxHex honeycomb consisting of auxetic and traditional honeycomb cells and studied the in-plane mechanical properties and energy absorption capability. Wu et al. [16] presented the plateau stresses and energy absorption capacity of a cross circular honeycombs with zero Poisson's ratio under in-plane impact loads. Wang et al. [17] gave effective elastic constants of a hierarchical composite squarehoneycomb core and studied free vibrations of the sandwich panel. Zhang et al. [18] investigated the equivalent macroscopic stiffness of honeycomb-corrugation hybrid cores and vibrations of the sandwich beams. These abovementioned references on the equivalent constants of the cores and vibrations of the honeycomb structures will provide guidance for this article.

It is known that avoiding flutter is one of the most important issues for aircraft design. Many research works are focused on the flutter responses of plates and shells because of recent wide use of advanced materials, and plentiful achievements have been obtained. Wang et al. [19] studied the flutter stability of a simply supported two-dimensional flat panel exposed to the supersonic airflow by using the first order piston theory aerodynamic loading. Guimarães et al. [20] investigated flutter of the supersonic adjacent composite panel through the first-order piston theory and compared the finite element model with Rayleigh-Ritz model. Song et al. [21] studied the panel flutter in supersonic airflow and passive control of the flutter bound. Mahmoudkhani [22] considered the supersonic panel flutter of functionally graded cylindrical shells under thermal loadings by using the firstorder aerodynamic piston theory. Dhital et al. [23] discussed the panel flutter whose model was based on the piston theory, where the aerodynamic forces were equivalent to the concentrated forces. Torabi et al. [24] analyzed free flexural vibration and supersonic flutter of trapezoidal honeycomb sandwich plates and aerodynamic pressure was estimated via the piston theory. Saidi et al. [25] probed the vibration and stability of functionally graded reinforced porous plates under supersonic flow. Zhang et al. [26,27] investigated nonlinear dynamics of plates under subsonic air flows. Hao et al. [28,29] gave supersonic flutter analysis of FGM shells through the first-order piston theory.

In this paper, free vibrations and flutter under supersonic airflows of a honeycomb sandwich plate with zero Poisson's ratio are analyzed. The theoretical model of the sandwich plate is obtained by using the Hamilton principle, higher order shear deformation theory and von Karman large geometric deformation theory in Section 2. The equivalent elastic parameters for the honeycomb core layer are computed in Section 3. The finite element model is given in Section 4. The first ten natural frequencies of the plate are obtained from both theory and finite element method. The consistency of the results from the two are compared which verify the correctness of the equivalent elastic parameters and the model obtained. The influence of the parameters on the natural frequency of the plate is obtained including the Poisson's ratio. In Section 5, flutters of the plate under supersonic airflows by using the first-order piston theory are analyzed and the change of the critical flutter point with the cell parameters of the zero Poisson's ratio honeycomb is obtained. In brief, the novelty of the paper is that: The equivalent elastic parameters for the ZPR honeycomb core are computed and their correctness is verified. When the Poisson's ratio of the honeycomb core is positive, zero and negative, respectively, the natural frequencies of the plates are compared. The ZPR honeycomb plate is used widely for aircraft skin, 
therefore, flutter of the ZPR honeycomb plate is studied and the critical flutter point of the plate is obtained in this paper.

The main symbols used in this paper are as follows: $u, v$ and $w$ are displacements in $x$, $y$ and $z$ directions, respectively. $\phi_{x}$ is rotation angle of the middle plane along $x$ direction. $a$, $b$ and $h$ are length, width and thickness of the plate. $\varepsilon$ and $\gamma$ are strains. $E, G, \rho$ and $v$ are Young's modulus, shear modulus, density and Poisson's ratio, the subscript ' $s$ ' represents the material. $\delta$ without subscript represents variational operation. $l_{1}, l_{2}, \mathrm{t}$ and $\theta$ are size and angle of the honeycomb cell. $n$ and $m$ are mode order of the plate.

\section{Equations of Motion}

The model of the honeycomb sandwich plate with zero Poisson's ratio under a foursided simply supported boundary is shown in Figure 1. The sandwich plate is composed of the honeycomb core with zero Poisson's ratio and two face sheets. The $x-y$ axis of coordinate system in Figure 1 is located on the middle plane of the plate. $u, v$ and $w$ represent the displacement components in $x, y$ and $z$ directions, respectively. The lengths of the plate in $x$ and $y$ directions are $a$ and $b$ respectively. The surface on one side of $z>0$ is called as upper skin, and the surface on the other side of $z<0$ is called as lower skin and the skin is made of isotropic materials. The material of the honeycomb plate is aluminum, whose modulus is $E_{s}$, density is $\rho_{s}$, Poisson's ratio is $v_{s}$, and shear modulus is $G_{s}$.

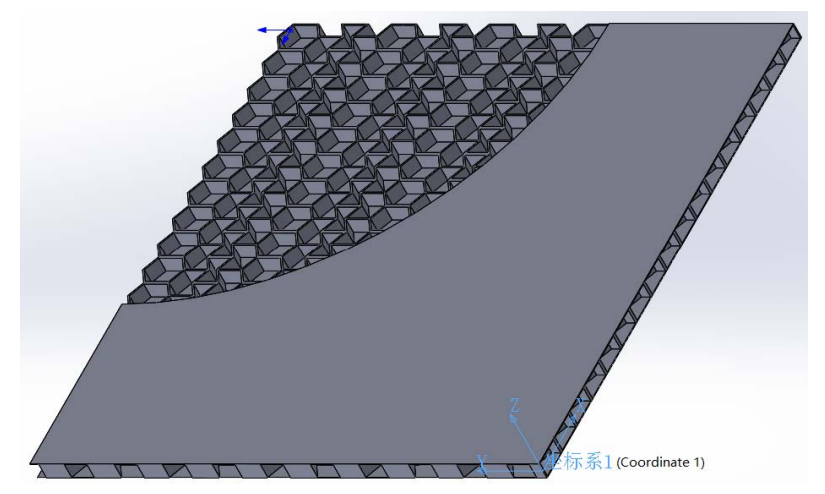

Figure 1. The model of honeycomb sandwich plate with ZPR core.

According to Reddy's higher order shear deformation theory, the displacements of $u$, $v$ and $w$ can be expressed as:

$$
\begin{gathered}
u(x, y, z, t)=u_{0}(x, y, t)+z \phi_{x}(x, y, t)-\frac{4}{3 h^{2}} z^{3}\left(\phi_{x}+\frac{\partial \omega_{0}}{\partial x}\right), \\
v(x, y, z, t)=v_{0}(x, y, t)+z \phi_{y}(x, y, t)-\frac{4}{3 h^{2}} z^{3}\left(\phi_{y}+\frac{\partial \omega_{0}}{\partial y}\right), \\
w(x, y, z, t)=w_{0}(x, y, t),
\end{gathered}
$$

where $h$ is the thickness of the plate, $u_{0}, v_{0}, w_{0}, \phi_{x}$ and $\phi_{y}$ are displacements and rotation angles of the middle plane. According to the von Karman large geometric deformation theory on the plate, the strain-displacement relations can be obtained as follows, where the strains are: $\varepsilon_{i}(i=x x, y y), \gamma_{i}(i=x y, y z, z x)$.

$$
\begin{gathered}
\varepsilon_{x x}=\frac{\partial u}{\partial x}+\frac{1}{2}\left(\frac{\partial w}{\partial x}\right)^{2}, \varepsilon_{y y}=\frac{\partial v}{\partial y}+\frac{1}{2}\left(\frac{\partial w}{\partial y}\right)^{2}, \gamma_{x z}=\frac{1}{2}\left(\frac{\partial u}{\partial z}+\frac{\partial w}{\partial x}\right), \\
\gamma_{y z}=\frac{1}{2}\left(\frac{\partial v}{\partial z}+\frac{\partial w}{\partial y}\right), \gamma_{x y}=\frac{\partial u}{\partial y}+\frac{\partial v}{\partial x}+\frac{\partial w}{\partial x} \frac{\partial w}{\partial y}, \varepsilon_{z z}=\frac{\partial w}{\partial z} .
\end{gathered}
$$

It can be obtained the following from Equations (1) and (2). 


$$
\left\{\begin{array}{c}
\varepsilon_{x x} \\
\varepsilon_{y y} \\
\gamma_{x y}
\end{array}\right\}=\left\{\begin{array}{c}
\varepsilon_{x x}^{(0)} \\
\varepsilon_{y y}^{(0)} \\
\gamma_{x y}^{(0)}
\end{array}\right\}+z\left\{\begin{array}{c}
\varepsilon_{x x}^{(1)} \\
\varepsilon_{y y}^{(1)} \\
\gamma_{x y}^{(1)}
\end{array}\right\}+z^{3}\left\{\begin{array}{c}
\varepsilon_{x x}^{(3)} \\
\varepsilon_{y y}^{(3)} \\
\gamma_{x y}^{(3)}
\end{array}\right\},\left\{\begin{array}{l}
\gamma_{y z} \\
\gamma_{x z}
\end{array}\right\}=\left\{\begin{array}{c}
(0) \\
\gamma_{y z}^{(0)} \\
\gamma_{x z}^{(0)}
\end{array}\right\}+z^{2}\left\{\begin{array}{c}
\gamma_{y z}^{(2)} \\
\gamma_{x z}^{(2)}
\end{array}\right\}
$$

The honeycomb sandwich plate with zero Poisson ratio is orthotropic and the constitutive equation can be written as:

$$
\left\{\begin{array}{c}
\sigma_{x x} \\
\sigma_{y y} \\
\sigma_{y z} \\
\sigma_{x z} \\
\sigma_{x y}
\end{array}\right\}=\left\{\begin{array}{ccccc}
Q_{11}^{(k)} & Q_{12}^{(k)} & 0 & 0 & 0 \\
Q_{21}^{(k)} & Q_{22}^{(k)} & 0 & 0 & 0 \\
0 & 0 & Q_{44}^{(k)} & 0 & 0 \\
0 & 0 & 0 & Q_{55}^{(k)} & 0 \\
0 & 0 & 0 & 0 & Q_{66}^{(k)}
\end{array}\right\}\left\{\begin{array}{c}
\varepsilon_{x x} \\
\varepsilon_{y y} \\
\gamma_{y z} \\
\gamma_{x z} \\
\gamma_{x y}
\end{array}\right\},
$$

where $Q_{11}^{(k)}=\frac{E_{1}^{(k)}}{1-v_{12}^{(k)} v_{21}^{(k)}}, Q_{12}^{(k)}=\frac{E_{1}^{(k)} v_{12}^{(k)}}{1-v_{12}^{(k)} v_{21}^{(k)}}, Q_{22}^{(k)}=\frac{E_{2}^{(k)}}{1-v_{12}^{(k)} v_{21}^{(k)}}, Q_{66}=G_{12}, Q_{44}=G_{23}$, $Q_{55}=G_{13}, Q_{21}=Q_{12}$, and $E_{1}^{(k)}, E_{2}^{(k)}, G_{12}^{(k)}, G_{13}^{(k)}, G_{23}^{(k)}, v_{12}^{(k)}, v_{21}^{(k)}$ are elastic modulus, shear modulus and Poisson's ratio, respectively, and $k=1,3$ represent the upper and lower skin, $k=2$ is the core layer.

The governing equations are established by using the Hamilton principle, and the expression of Hamilton principle is as follows:

$$
\int_{t_{1}}^{t_{2}}(\delta U-\delta K) d t+\int_{t_{1}}^{t_{2}} \delta W d t=0
$$

where $\delta U$ is the virtual potential energy, $\delta K$ is the virtual kinetic energy, and $\delta W$ is the virtual work done by external forces. The virtual kinetic energy and virtual potential energy can be casted in the following equations:

$$
\begin{gathered}
\delta K=\int_{\Omega_{0}}\left[\left(I_{0} \dot{u}_{0}+I_{1} \dot{\phi}_{x}-c_{1} I_{3} \dot{\varphi}_{x}\right) \delta \dot{u}_{0}+\left(I_{1} \dot{u}_{0}+I_{2} \dot{\phi}_{x}-c_{1} I_{4} \dot{\varphi}_{x}\right) \delta \dot{\phi}_{x}\right. \\
-c_{1}\left(I_{3} \dot{u}_{0}+I_{4} \dot{\phi}_{x}-c_{1} I_{6} \dot{\varphi}_{x}\right) \delta \dot{\varphi}_{x}+\left(I_{0} \dot{v}_{0}+I_{1} \dot{\phi}_{y}-c_{1} I_{3} \dot{\varphi}_{y}\right) \delta \dot{v}_{0}+\left(I_{1} \dot{v}_{0}+I_{2} \dot{\phi}_{y}-c_{1} I_{4} \dot{\varphi}_{y}\right) \delta \dot{\phi}_{y} \\
\left.-c_{1}\left(I_{3} \dot{v}_{0}+I_{4} \dot{\phi}_{y}-c_{1} I_{6} \dot{\varphi}_{y}\right) \delta \dot{\varphi}_{y}-I_{0} \dot{w}_{0} \delta \dot{w}_{0}\right] d x d y,
\end{gathered}
$$

where

$$
\begin{gathered}
\dot{\varphi}_{x}=\dot{\phi}_{x}+\frac{\partial w_{0}}{\partial x}, \dot{\varphi}_{y}=\dot{\phi}_{y}+\frac{\partial w_{0}}{\partial y} \\
\delta U=\int_{\Omega_{0}}\left[N_{x x} \delta \varepsilon_{x x}^{(0)}+M_{x x} \delta \varepsilon_{x x}^{(1)}-c_{1} P_{x x} \delta \varepsilon_{x x}^{(3)}+N_{y y} \delta \varepsilon_{y y}^{(0)}+M_{y y} \delta \varepsilon_{y y}^{(1)}\right. \\
-c_{1} P_{y y} \delta \varepsilon_{y y}^{(3)}+N_{x y} \delta \gamma_{x y}^{(0)}+M_{x y} \delta \gamma_{x y}^{(1)}-c_{1} P_{x y} \delta \gamma_{x y}^{(3)}+Q_{x} \delta \gamma_{x z}^{(0)} \\
\left.-c_{2} R_{x} \delta \gamma_{x z}^{(0)}+Q_{y} \delta \gamma_{y z}^{(0)}-c_{2} R_{y} \delta \gamma_{y z}^{(0)}\right] d x d y, \\
\delta W=\int_{\Omega_{0}} F \delta w_{0} d x d y-\int_{\Omega_{0}} \gamma \delta \dot{w}_{0} d x d y .
\end{gathered}
$$

$\Omega_{0}$ represents the middle plane of the honeycomb sandwich plate, $\gamma$ is the damping coefficient and

$$
\begin{gathered}
\left\{\begin{array}{c}
N_{\alpha \beta} \\
M_{\alpha \beta} \\
P_{\alpha \beta}
\end{array}\right\}=\int_{-\frac{h}{2}}^{\frac{h}{2}} \sigma_{\alpha \beta}\left\{\begin{array}{c}
1 \\
z \\
z^{3}
\end{array}\right\} d z, \\
\left\{\begin{array}{c}
Q_{\alpha} \\
R_{\alpha}
\end{array}\right\}=\int_{-\frac{h}{2}}^{\frac{h}{2}} \sigma_{\alpha z}\left\{\begin{array}{c}
1 \\
z^{2}
\end{array}\right\} d z I_{i}=\int_{-\frac{h}{2}}^{\frac{h}{2}} \rho_{s}(z)^{i} d z,(i=0,1,2, \cdots, 6),
\end{gathered}
$$

where $\alpha$ and $\beta$ represent $x$ and $y$, respectively. 
Substituting Equations (6), (8) and (9) into Equation (5), the nonlinear dynamic equation of the honeycomb sandwich plate can be written:

$$
\begin{gathered}
\frac{\partial N_{x x}}{\partial x}+\frac{\partial N_{x y}}{\partial y}=I_{0} \ddot{u}_{0}+J_{1} \ddot{\phi}_{x}-c_{1} I_{3} \frac{\partial \ddot{w}_{0}}{\partial x}, \\
\frac{\partial N_{x y}}{\partial x}+\frac{\partial N_{y y}}{\partial y}=I_{0} \ddot{v}_{0}+J_{1} \ddot{\phi}_{y}-c_{1} I_{3} \frac{\partial \ddot{w}_{0}}{\partial y}, \\
\frac{\partial \bar{Q}_{x}}{\partial x}+\frac{\partial \bar{Q}_{y}}{\partial y}+\frac{\partial}{\partial x}\left(N_{x x} \frac{\partial w_{0}}{\partial x}+N_{x y} \frac{\partial w_{0}}{\partial y}\right)+\frac{\partial}{\partial y}\left(N_{x y} \frac{\partial w_{0}}{\partial x}+N_{y y} \frac{\partial w_{0}}{\partial y}\right)+c_{1}\left(\frac{\partial^{2} P_{x x}}{\partial x^{2}}+2 \frac{\partial^{2} P_{x y}}{\partial x \partial y}+\frac{\partial^{2} P_{y y}}{\partial y^{2}}\right) \\
=I_{0} \ddot{w}_{0}-c_{1}^{2} I_{6}\left(\frac{\partial^{2} \ddot{w}_{0}}{\partial x^{2}}+\frac{\partial^{2} \ddot{w}_{0}}{\partial y^{2}}\right)+c_{1}\left[I_{3}\left(\frac{\partial \ddot{u}_{0}}{\partial x}+\frac{\partial \ddot{w}_{0}}{\partial y}\right)+J_{4}\left(\frac{\partial \ddot{\phi}_{x}}{\partial x}+\frac{\partial \ddot{\phi}_{y}}{\partial y}\right)\right], \\
\frac{\partial \bar{M}_{x x}}{\partial x}+\frac{\partial \bar{M}_{x y}}{\partial y}-\bar{Q}_{x}=J_{1} \ddot{u}_{0}+K_{2} \ddot{\phi}_{x}-c_{1} J_{4} \frac{\partial \ddot{w}_{0}}{\partial x}, \\
\frac{\partial \bar{M}_{x y}}{\partial x}+\frac{\partial \bar{M}_{y y}}{\partial y}-\bar{Q}_{y}=J_{1} \ddot{v}_{0}+K_{2} \ddot{\phi}_{y}-c_{1} J_{4} \frac{\partial \ddot{w}_{0}}{\partial y},
\end{gathered}
$$

where $\bar{M}_{\alpha \beta}=M_{\alpha \beta}-c_{1} P_{\alpha \beta},(\alpha, \beta=1,2,6), \bar{Q}_{\alpha}=Q_{\alpha}-c_{2} R_{\alpha},(\alpha=4,5), J_{i}=I_{i}-c_{1} I_{i+2}$, $K_{2}=I_{2}-2 c_{1} I_{4}+c_{1}^{2} I_{6} c_{1}=\frac{4}{3 h^{2}}, c_{2}=3 c_{1}$.

The relationship of internal force and strain is of the following form:

$$
\begin{gathered}
\left\{\begin{array}{l}
N_{x x} \\
N_{y y} \\
N_{x y}
\end{array}\right\}=\left\{\begin{array}{ccc}
A_{11} & A_{12} & 0 \\
A_{21} & A_{22} & 0 \\
0 & 0 & A_{66}
\end{array}\right\}\left\{\begin{array}{c}
\varepsilon_{x x}^{(0)} \\
\varepsilon_{y y}^{(0)} \\
\gamma_{x y}^{(0)}
\end{array}\right\}, \\
\left\{\begin{array}{l}
M_{x x} \\
M_{y y} \\
M_{x y}
\end{array}\right\}=\left\{\begin{array}{ccc}
D_{11} & D_{12} & 0 \\
D_{21} & D_{22} & 0 \\
0 & 0 & D_{66}
\end{array}\right\}\left\{\begin{array}{l}
\varepsilon_{x x}^{(1)} \\
\varepsilon_{y y}^{(1)} \\
\gamma_{x y}^{(1)}
\end{array}\right\}+\left\{\begin{array}{ccc}
F_{11} & F_{12} & 0 \\
F_{21} & F_{22} & 0 \\
0 & 0 & F_{66}
\end{array}\right\}\left\{\begin{array}{l}
\varepsilon_{x x}^{(3)} \\
\varepsilon_{y y}^{(3)} \\
\gamma_{x y}^{(3)}
\end{array}\right\}, \\
\left\{\begin{array}{l}
P_{x x} \\
P_{y y} \\
P_{x y}
\end{array}\right\}=\left\{\begin{array}{ccc}
F_{11} & F_{12} & 0 \\
F_{21} & F_{22} & 0 \\
0 & 0 & F_{66}
\end{array}\right\}\left\{\begin{array}{l}
\varepsilon_{x x}^{(1)} \\
\varepsilon_{y y}^{(1)} \\
\gamma_{x y}^{(1)}
\end{array}\right\}+\left\{\begin{array}{ccc}
H_{11} & H_{12} & 0 \\
H_{21} & H_{22} & 0 \\
0 & 0 & H_{66}
\end{array}\right\}\left\{\begin{array}{l}
\varepsilon_{x x}^{(3)} \\
\varepsilon_{y y}^{(3)} \\
\gamma_{x y}^{(3)}
\end{array}\right\}, \\
\left\{\begin{array}{l}
Q_{y} \\
Q_{x}
\end{array}\right\}=\left\{\begin{array}{cc}
A_{44} & 0 \\
0 & A_{55}
\end{array}\right\}\left\{\begin{array}{l}
\gamma_{y z}^{(0)} \\
\gamma_{x z}^{(0)}
\end{array}\right\}+\left\{\begin{array}{cc}
D_{44} & 0 \\
0 & D_{55}
\end{array}\right\}\left\{\begin{array}{l}
\gamma_{y z}^{(2)} \\
\gamma_{x z}^{(2)}
\end{array}\right\}, \\
\left\{\begin{array}{l}
R_{y} \\
R_{x}
\end{array}\right\}=\left\{\begin{array}{cc}
D_{44} & 0 \\
0 & D_{55}
\end{array}\right\}\left\{\begin{array}{l}
\gamma_{y z}^{(0)} \\
\gamma_{x z}^{(0)}
\end{array}\right\}+\left\{\begin{array}{cc}
F_{44} & 0 \\
0 & F_{55}
\end{array}\right\}\left\{\begin{array}{l}
(2) \\
\gamma_{y z}^{(2)} \\
\gamma_{x z}^{(2)}
\end{array}\right\},
\end{gathered}
$$

where the stiffness matrices of the plates are expressed as follows:

$$
\begin{gathered}
\left(A_{i j}, D_{i j}, F_{i j}, H_{i j}\right)=\int_{-h / 2}^{h / 2} Q_{i j}\left(1, z^{2}, z^{4}, z^{6}\right) d z,(i, j=1,2,6), \\
\left(A_{i j}, D_{i j}, F_{i j}\right)=\int_{-h / 2}^{h / 2} Q_{i j}\left(1, z^{2}, z^{4}\right) d z,(i, j=4,5),
\end{gathered}
$$

By substituting Equation (12) into Equation (11), the nonlinear dynamic equation of honeycomb sandwich plate with zero Poisson's ratio in the form of displacement can be obtained.

\section{Calculation of Equivalent Elastic Parameters}

The honeycomb cells of zero Poisson's ratio are composed of regular hexagonal mixed with concave hexagonal honeycombs as shown in Figure 2. These kinds of mixed honeycombs exhibit zero Poisson's ratio due to the positive Poisson's ratio effect of the 
hexagonal honeycomb and the negative Poisson's ratio effect of the concave hexagonal honeycomb [30].

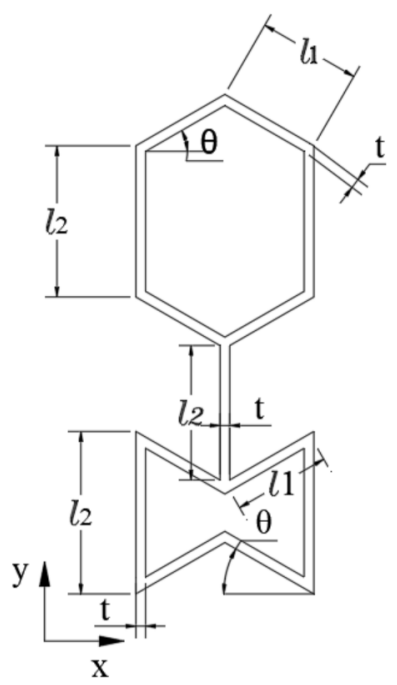

Figure 2. Honeycomb cell with zero Poisson's ratio.

In the literature [14], the in-plane mechanical properties of hybrid AuxHex honeycomb are derived and analytical formulas of the relative density and Young's modulus are given. For simplification, it is considered that the inclined wall and straight wall of the hybrid honeycombs have the same length and thickness and same angles as shown in Figure 2.

Relative density is an important performance index for honeycomb materials which is referred to the ratio of the density of porous materials to that of the corresponding solid materials. As shown in Figure 2, the relative density of the honeycomb with zero Poisson's ratio is as follows:

$$
\bar{\rho}=\frac{\rho^{*}}{\rho_{s}}=\frac{8 l_{1} t+5 l_{2} t}{6 l_{1} l_{2} \cos \theta}
$$

where $\rho^{*}$ and $\rho_{s}$ densities of honeycombs and the materials, respectively. The Young's modulus of the honeycombs is given as follows:

$$
E_{x}=\frac{\sigma_{1}}{\varepsilon_{x}^{x}}=\frac{E_{s} t^{3} \cos \theta}{l_{2} l_{1}^{2} \sin ^{2} \theta}, \quad E_{y}=\frac{\sigma_{2}}{\varepsilon_{y}^{y}}=\frac{2 E_{s} t^{3} l_{2}}{l_{1}^{4} \cos ^{3} \theta},
$$

where the superscript $x$ or $y$ of the strain $\varepsilon$ indicates the corresponding strain when loading in $x$ or $y$ direction, respectively. $\sigma_{1}$ and $\sigma_{2}$ are stresses as shown in Figure 3.

The Poisson's ratio is computed as follows. When loading in the $x$-axis, as seen in Figure $3 a$, the strain of wall $A B$ in the $y$-direction is:

$$
\varepsilon_{h y}^{x}=\frac{2 \delta_{h} \cos \theta}{2 l_{2}+2 l_{1} \sin \theta}=\frac{\sigma_{1}}{E_{s}} \frac{l_{1}^{3} \sin \theta \cos \theta}{t^{3}},
$$

where $\delta_{h}$ is the deformation of wall $\mathrm{AB}$, as observed in the literature [14]. The strain of the wall $C D$ in y-direction is:

$$
\varepsilon_{a y}^{x}=-\frac{2 \delta_{a} \cos \theta}{2 l_{2}-2 l_{1} \sin \theta}=-\frac{\sigma_{1}}{E_{s}} \frac{l_{1}^{3} \sin \theta \cos \theta}{t^{3}},
$$

where $\delta_{a}$ is deformation of the wall CD. Compared with Equations (16) and (17), the strains of regular and concave hexagon in the y direction, as shown in Figure $3 a$, are equal and 
opposite, that is, the strain in the $y$-direction is $\varepsilon_{y}^{x}=0$. Therefore, the Poisson's ratio in the $x$-direction is:

$$
v_{x y}=-\frac{\varepsilon_{y}^{x}}{\varepsilon_{x}^{x}}=0,
$$

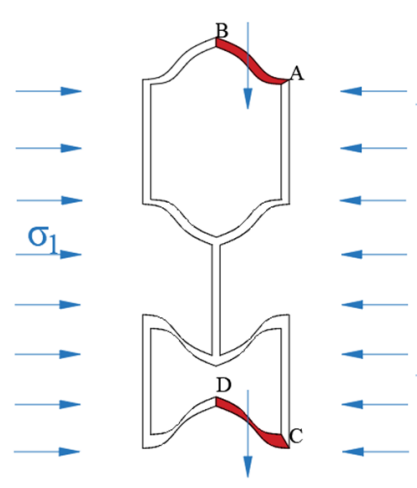

(a)

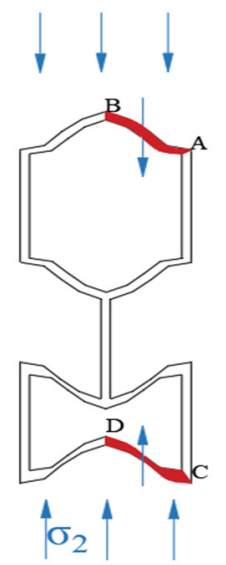

(b)

Figure 3. Uniaxial compressive of the honeycombs. (a) $x$-axis loading, (b) $y$-axis loading.

When loading in $y$-axis as in Figure $3 b$, the strain of wall $\mathrm{AB}$ in the $x$-direction is:

$$
\varepsilon_{h x}^{y}=\frac{\delta_{h} \sin \theta}{l_{1} \cos \theta}=\frac{\sigma_{2} l_{1}^{3} \cos \theta \sin \theta}{E_{s} t^{3}}
$$

The strain of wall CD in the $x$-direction is:

$$
\varepsilon_{a x}^{y}=-\frac{\delta_{a} \sin \theta}{l_{1} \cos \theta}=-\frac{\sigma_{2} l_{1}^{3} \cos \theta \sin \theta}{E_{s} t^{3}}
$$

where $\delta_{h}$ and $\delta_{a}$ are deformations of walls $\mathrm{AB}$ and $\mathrm{CD}$ in Figure $3 \mathrm{~b}$, that is, the strain in the $x$-direction is:

$$
\varepsilon_{x}^{y}=\frac{\varepsilon_{h x}^{y}+\varepsilon_{a x}^{y}}{2}=0
$$

Therefore, the Poisson's ratio in y-direction is:

$$
v_{y x}=-\frac{\varepsilon_{x}^{y}}{\varepsilon_{y}^{y}}=0
$$

Therefore, it is proved that the Poisson's ratio of honeycombs as shown in Figure 2 is zero when the angles of regular hexagonal and concave hexagonal honeycombs are same.

Next, the shear modulus is given. It is based on that the strain energy of the zero Poisson's ratio cell by shear loads is equal to the sum of the strain energy of a regular hexagonal and a concave hexagonal honeycomb cell, the shear modulus $G_{x y}$ can be obtained [30]:

$$
G_{x y}=\frac{2 \alpha G_{h x y} G_{a x y}}{(\alpha+\sin \theta) G_{h x y}+(\alpha-\sin \theta) G_{a x y}},
$$

where $G_{h x y}$ and $G_{a x y}$ are shear modulus of regular hexagonal and concave hexagonal honeycomb, and $\alpha=\frac{l_{2}}{l_{1}}$. The shear modulus of the other two directions are obtained by principle of minimum energy $[30,31]$.

$$
G_{z x}=G_{s} \frac{\beta \cos \theta}{\alpha}
$$




$$
G_{s} \beta \frac{(\alpha+\sin \theta)^{2}}{\alpha(1+2 \alpha) \cos \theta} \leq G_{y z} \leq G_{s} \beta \frac{\alpha+2 \sin ^{2} \theta}{2 \alpha \cos \theta},
$$

where $G_{s}$ is shear modulus of materials and $\beta=\frac{t}{l_{1}}$, it can be obtained by calculation from Equation (24b) that the difference between upper and lower of $G_{y z}$ is small, which has less effect on the vibrational frequency of the honeycomb.

Substitute the equivalent elastic parameters given above into Equation (4), where the subscripts $x$ and $y$ in Equations (15)-(24) are replaced by 1 and 2 in Equation (4). Then solve the zero Poisson's ratio honeycomb sandwich plate (11) by the Navier method and compare the results with the finite element model in next section.

\section{Vibrational Frequencies of ZPR Honeycomb Plate}

The first ten $(n=1,2 \cdots, 10)$ natural frequencies of the honeycomb sandwich plate with zero Poisson's ratio are obtained by using the Navier method and through the finite element model simultaneously. The finite element models are obtained by using ABAQUS/Standard. A solid model is adopted, and each cell wall of honeycombs is solved by a four-node linear tetrahedral element grid. The boundary conditions in the modal analysis settled as simply supported on four sides, and the ABAQUS/standard subspace iteration method is used to solve the natural frequency of the system.

The parameters of the honeycomb plate are chosen as same in the two methods as: The length of the plate is $a=311 \mathrm{~mm}$, the width is $b=311 \mathrm{~mm}$, the thickness of the plate $h=$ $10 \mathrm{~mm}$, the thickness of the core layer $h_{c}=9 \mathrm{~mm}$, the parameters of honeycomb cell $l_{1}=10$ $\mathrm{mm}, t=1 \mathrm{~mm}, l_{2}=12 \mathrm{~mm}, \theta=\pi / 6$ as shown in Figure 2. The plate is made of aluminum, the Young's modulus is $E_{s}=69 \mathrm{GPa}$, the density $\rho_{s}=2700 \mathrm{~kg} / \mathrm{m}^{3}$, the Poisson's ratio $v_{s}=$ 0.3 , the shear modulus $G_{s}=27 \mathrm{GPa}$.

Table 1 shows the first ten natural frequencies of the honeycomb sandwich plate with zero Poisson's ratio by using the Navier method and the finite element method. The errors between the two methods are given. From the comparison results in Table 1 it can be found that the maximum error from Section 3 and the finite element method is $1.02 \%$, and the maximum error between the literature [14] and the finite element method is $1.55 \%$. The maximum calculation error is smaller in this paper. The reason is that the concave hexagon only was calculated when loading in the $y$ direction the literature [14], that is the wall $\mathrm{CD}$ in Figure 3b. Furthermore, it is inconvenient to calculate the Poisson's ratio, while the difference between the two is not so big, which indicates that the concave hexagonal honeycombs play a main role when loading in the $y$ direction. However, the regular hexagonal honeycombs in the hybrid honeycombs have great influence on the Poisson's ratio of the honeycomb structures. Table 1 also shows the validity of the equivalent elastic parameters for the honeycomb core layer and the theoretical model of the plate.

Table 1. The first ten natural frequencies of the honeycomb sandwich plate with ZPR.

\begin{tabular}{cccccc}
\hline $\mathbf{n}$ & FEM & This Paper & Error & Ref. [14] & Error \\
\hline 1 & 550.27 & 546.43 & $0.70 \%$ & 547.27 & $0.54 \%$ \\
2 & 1358.0 & 1344.01 & $1.02 \%$ & 1347.44 & $0.78 \%$ \\
3 & 1360.9 & 1349.11 & $0.86 \%$ & 1349.84 & $0.81 \%$ \\
4 & 2114.6 & 2123.63 & $-0.42 \%$ & 2126.64 & $-0.57 \%$ \\
5 & 2642.5 & 2621.39 & $0.79 \%$ & 2628.63 & $0.52 \%$ \\
6 & 2654.5 & 2641.72 & $0.48 \%$ & 2642.37 & $0.46 \%$ \\
7 & 3334.8 & 3366.87 & $-0.96 \%$ & 3384.45 & $-1.49 \%$ \\
8 & 3350.9 & 3381.82 & $-0.92 \%$ & 3373.42 & $-0.67 \%$ \\
9 & 4299.8 & 4312.93 & $-0.30 \%$ & 4366.38 & $-1.55 \%$ \\
10 & 4353.6 & 4365.81 & $-0.28 \%$ & 4324.51 & $0.67 \%$ \\
\hline
\end{tabular}

Table 2 shows the first ten order natural frequencies of the plate with the hexagonal honeycomb core and concave hexagonal honeycomb core respectively, with the same parameters chosen as those in Table 1. Figure 4 compares the three kinds of plates with 
the Poisson's ratios of the honeycomb cores that are positive, zero and negative. It can be obtained from Figure 4 that, with the other parameters are all same, the frequencies of the auxetic honeycomb sandwich plate are low, the frequencies of the regular hexagon cores are high and the hybrids' (ZPR) are middle. The difference between the hexagon and the hybrids is small while the auxetic is much lower.

Table 2. The frequencies of the plate with regular hexagonal and concave hexagonal cores.

\begin{tabular}{ccccc}
\hline $\mathbf{n}$ & \multicolumn{2}{c}{ Hexagonal Honeycomb } & \multicolumn{2}{c}{ Auxetic Honeycomb } \\
\cline { 2 - 5 } & FEM & Theoretical & FEM & Theoretical \\
\hline 1 & 562.41 & 561.48 & 467.76 & 453.47 \\
2 & 1384.7 & 1379.67 & 1163.5 & 1107.25 \\
3 & 1403.3 & 1423.76 & 1184.5 & 1158.23 \\
4 & 2182.7 & 2173.02 & 1835.4 & 1775.68 \\
5 & 2710.6 & 2699.06 & 2304.5 & 2172.77 \\
6 & 2755.9 & 2814.52 & 2366.7 & 2311.28 \\
7 & 3450.0 & 3424.70 & 2932.7 & 2803.57 \\
8 & 3496.9 & 3498.36 & 2976.2 & 2892.68 \\
9 & 4459.6 & 4432.39 & 3840.3 & 3600.79 \\
10 & 4689.9 & 4645.49 & 3973.6 & 3867.33 \\
\hline
\end{tabular}

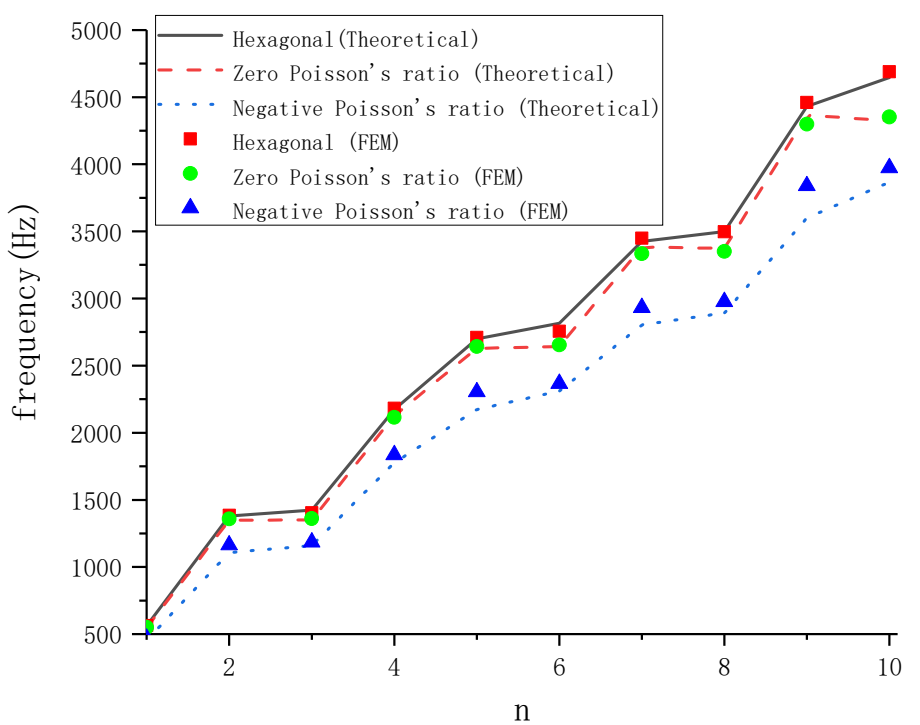

Figure 4. Comparison of the frequencies of three honeycomb sandwich plate.

When the core thickness changes, the thickness of the sandwich plate invariant and other parameters are chosen as the same as that of Table 1, and Table 3 shows the first ten order frequencies. It can be obtained from Table 3 that when the thickness of the core layer increases and the thickness of the plate is invariant, the thickness of the skin decreases, and the natural frequency of the zero Poisson's ratio honeycomb sandwich plate decreases. 
Table 3. Frequencies of ZPR honeycomb plate with core thickness ratio change.

\begin{tabular}{ccccc}
\hline $\mathbf{n}$ & $\boldsymbol{h}_{\boldsymbol{c}} / \boldsymbol{h}=\mathbf{0 . 8}$ & $\boldsymbol{h}_{\boldsymbol{c}} / \boldsymbol{h}=\mathbf{0 . 8 5}$ & $\boldsymbol{h}_{\boldsymbol{c}} / \boldsymbol{h}=\mathbf{0 . 9}$ & $\boldsymbol{h}_{\boldsymbol{c}} / \boldsymbol{h}=\mathbf{0 . 9 5}$ \\
\hline 1 & 617.79 & 594.253 & 547.27 & 446.52 \\
2 & 1513.17 & 1459.26 & 1347.44 & 1107.69 \\
3 & 1508.57 & 1455.44 & 1349.84 & 1107.66 \\
4 & 2366.57 & 2288.43 & 2126.64 & 1756.13 \\
5 & 2908.48 & 2837.38 & 2628.63 & 2188.04 \\
6 & 2930.31 & 2817.76 & 2642.37 & 2184.33 \\
7 & 3712.80 & 3619.52 & 3384.45 & 2814.48 \\
8 & 3729.46 & 3604.22 & 3373.42 & 2818.07 \\
9 & 4718.41 & 4650.07 & 4366.38 & 3641.42 \\
10 & 4778.78 & 4593.51 & 4324.51 & 3656.91 \\
\hline
\end{tabular}

Table 4 shows that the frequencies with the length of honeycomb wall $l_{2}$ change, while the other parameters remain unchanged. It can be seen that the smaller the cell wall length, that is the tighter the cell arrangement, the lower the frequency of the plate is.

Table 4. Frequencies of ZPR honeycomb plate with $l_{2}$ change.

\begin{tabular}{cccc}
\hline $\mathbf{n}$ & $\boldsymbol{l}_{\mathbf{2}} \mathbf{=} \mathbf{1 2} \mathbf{~} \mathbf{m}$ & $\boldsymbol{l}_{\mathbf{2}}=\mathbf{1 5} \mathbf{~ \mathbf { m }}$ & $\boldsymbol{l}_{\mathbf{2}} \mathbf{=} \mathbf{2 0} \mathbf{~ \mathbf { m }}$ \\
\hline 1 & 547.27 & 562.33 & 579.62 \\
2 & 1347.44 & 1382.69 & 1418.19 \\
3 & 1349.84 & 1385.26 & 1429.00 \\
4 & 2126.64 & 2179.11 & 2236.40 \\
5 & 2628.63 & 2695.28 & 2746.12 \\
6 & 2642.37 & 2702.48 & 2788.19 \\
7 & 3384.45 & 3453.04 & 3520.05 \\
8 & 3373.42 & 3457.65 & 3550.66 \\
9 & 4366.38 & 4430.81 & 4478.51 \\
10 & 4324.51 & 4445.05 & 4585.30 \\
\hline
\end{tabular}

With the cell angle $\theta$ changed to $15^{\circ}, 30^{\circ}, 45^{\circ}$, and $60^{\circ}$, while the other parameters are the same as that of above, Table 5 shows the frequencies for the first ten order modes. It is indicated that the natural frequency of the plate decreases with angle $\theta$ increases.

Table 5. Frequencies of ZPR honeycomb plate with angle $\theta$ change.

\begin{tabular}{|c|c|c|c|c|}
\hline n & $\theta=15^{\circ}$ & $\theta=30^{\circ}$ & $\theta=45^{\circ}$ & $\theta=60^{\circ}$ \\
\hline 1 & 605.06 & 579.62 & 538.14 & 472.27 \\
\hline 2 & 1487.16 & 1418.19 & 1309.53 & 1134.05 \\
\hline 3 & 1482.24 & 1429.00 & 1336.45 & 1188.85 \\
\hline 4 & 2328.62 & 2236.40 & 2078.65 & 1818.49 \\
\hline 5 & 2889.36 & 2746.12 & 2521.17 & 2156.99 \\
\hline 6 & 2872.36 & 2788.19 & 2629.03 & 2362.50 \\
\hline 7 & 3680.81 & 3520.05 & 3329.36 & 2801.11 \\
\hline 8 & 3668.92 & 3550.66 & 3250.10 & 2949.38 \\
\hline 9 & 4726.80 & 4478.51 & 4085.16 & 3449.49 \\
\hline 10 & 4686.85 & 4585.30 & 4364.08 & 3959.42 \\
\hline
\end{tabular}

Table 6 shows that the frequencies of the honeycomb sandwich with zero Poisson's ratio decrease when the thickness of the honeycomb wall increase. 
Table 6. Frequencies with thickness of honeycomb wall change.

\begin{tabular}{ccccc}
\hline $\mathbf{n}$ & $\boldsymbol{t}$ & $\boldsymbol{t}$ & $\boldsymbol{t}$ & $\boldsymbol{t}$ \\
\hline 1 & 546.43 & 488.75 & 448.70 & 420.77 \\
2 & 1344.01 & 1211.28 & 1115.74 & 1049.86 \\
3 & 1349.11 & 1211.53 & 1119.13 & 1057.31 \\
4 & 2123.63 & 1914.56 & 1764.74 & 1658.71 \\
5 & 2621.39 & 2382.99 & 2209.22 & 2087.67 \\
6 & 2641.72 & 2387.43 & 2214.02 & 2103.45 \\
7 & 3366.87 & 3060.01 & 2833.65 & 2669.13 \\
8 & 3381.82 & 3064.55 & 2835.19 & 2677.72 \\
9 & 4312.93 & 3956.04 & 3695.20 & 3504.64 \\
10 & 4365.81 & 3976.10 & 3696.12 & 3524.73 \\
\hline
\end{tabular}

Table 7 shows that the frequencies of the honeycomb sandwich with zero Poisson's ratio decrease when the length-width of the plate increase.

Table 7. Frequencies with length-width change.

\begin{tabular}{cccccc}
\hline $\boldsymbol{n}$ & $\boldsymbol{b} / \boldsymbol{a}=\mathbf{1}$ & $\boldsymbol{b} / \boldsymbol{a}=\mathbf{1 . 5}$ & $\boldsymbol{b} / \boldsymbol{a}=\mathbf{2}$ & $\boldsymbol{b} / \boldsymbol{a}=\mathbf{2 . 5}$ & $\boldsymbol{b} / \boldsymbol{a}=\mathbf{3}$ \\
\hline 1 & 546.43 & 396.43 & 343.46 & 318.92 & 305.59 \\
2 & 1344.01 & 757.08 & 547.27 & 449.61 & 396.43 \\
3 & 1349.11 & 1203.57 & 883.66 & 666.17 & 547.27 \\
4 & 2123.63 & 1347.44 & 1152.21 & 966.43 & 757.08 \\
5 & 2621.39 & 1553.36 & 1347.44 & 1128.40 & 1024.41 \\
6 & 2641.72 & 2126.64 & 1349.84 & 1255.14 & 1115.46 \\
7 & 3366.87 & 2151.97 & 1676.20 & 1347.44 & 1203.57 \\
8 & 3381.82 & 2502.77 & 1931.76 & 1465.16 & 1347.44 \\
9 & 4312.93 & 2836.67 & 2126.64 & 1756.54 & 1349.84 \\
10 & 4365.81 & 2909.19 & 2453.74 & 2126.64 & 1553.36 \\
\hline
\end{tabular}

In this section, the model of the honeycomb sandwich plate with zero Poisson's ratio is obtained through both the theoretical method and finite element method, which proves the accuracy of the equivalent elastic parameters of the honeycomb core layer and the validity of the model. The influence of the geometric parameters of the plate on the natural frequencies is studied.

\section{Flutter Analysis under Aerodynamic Forces}

The zero-Poisson's-ratio honeycomb sandwich plate has potential applications in wing skins because it has no Poisson's ratio effect during the deformation process. Large deformations introduce considerable aeroelastic effects. Moreover, the aeroelastic instability of the plate is a prominent problem that can be devastating to the structures. Therefore, it is very necessary to study the flutter problem of the zero-Poisson's-ratio honeycomb sandwich plate. When the plate is under supersonic airflow, based on the first-order piston theory, the aerodynamic pressure load is expressed as:

$$
D P=-\frac{2 P_{\infty}}{\sqrt{M_{a}^{2}-1}}\left(\frac{M_{a}^{2}-2}{M_{a}^{2}-1} \frac{1}{M_{a} a_{\infty}} \frac{\partial w}{\partial t}+\frac{\partial w}{\partial x}\right),
$$

where $P_{\infty}=\frac{1}{2} \rho_{\infty} U_{\infty}^{2}$ is flow pressure, $U_{\infty}$ is air velocity at infinity, $a_{\infty}, M_{a}$ and $\rho_{\infty}$ are sound airspeed, Mach number and air density respectively. Substitute aerodynamic pressure load (25) into the external force work expression in Equation (9). Meanwhile, Take the modal functions (26) that satisfy the four-sided simply supported boundary and substitute them into Equation (11) by using the Galerkin method.

$$
u_{0}=\sum_{m=1}^{M} \sum_{n=1}^{N} u_{m n}(t) \cos \left(\frac{m \pi x}{a}\right) \sin \left(\frac{n \pi y}{b}\right),
$$




$$
\begin{aligned}
& v_{0}=\sum_{m=1}^{M} \sum_{n=1}^{N} v_{m n}(t) \sin \left(\frac{m \pi x}{a}\right) \cos \left(\frac{n \pi y}{b}\right), \\
& w_{0}=\sum_{m=1}^{M} \sum_{n=1}^{N} w_{m n}(t) \sin \left(\frac{m \pi x}{a}\right) \cos \left(\frac{n \pi y}{b}\right), \\
& \phi_{x}=\sum_{m=1}^{M} \sum_{n=1}^{N} \phi_{x m n}(t) \cos \left(\frac{m \pi x}{a}\right) \sin \left(\frac{n \pi y}{b}\right), \\
& \phi_{y}=\sum_{m=1}^{M} \sum_{n=1}^{N} \phi_{y m n}(t) \sin \left(\frac{m \pi x}{a}\right) \cos \left(\frac{n \pi y}{b}\right),
\end{aligned}
$$

where $u_{m n}, v_{m n}, w_{m n}, \phi_{x m n}$ and $\phi_{y m n}$ are the amplitudes of each mode. Neglect the inertial terms of $u_{0}, v_{0}, \phi_{x}$ and $\phi_{y}$ in Equation (11) and substitute into Equation (11c), it can be obtained the equation of motion for the honeycomb sandwich plate with zero Poisson's ratio under aerodynamic pressure load as Equation (27).

$$
\ddot{w}_{m n}(t)+\left(\mu_{k m n}+\mu_{a m n}\right) \dot{w}_{m n}(t)+\left(C_{k m n}+C_{a m n}\right) w_{m n}(t)+V\left(w_{m n}(t)\right)=0,
$$

where the subscripts " $k$ " and " $a$ " in $\mu$ and $C$ represent coefficients of structural and aerodynamic terms, $V$ represents nonlinear terms. Assuming that the airflow enters along the $x$-axis, vertical to the $y$ direction. It is known that the higher-order modes perpendicular to the airflow direction are difficult to be excited under the condition of the simply supported boundary, therefore, it is taken the first mode in the $y$ direction, that is $N=1$.

Suppose Equation (27) has solutions of the form:

$$
w_{m}=A_{m} e^{\lambda t}
$$

where $\lambda=\lambda_{r}+i \lambda_{i}, \lambda_{r}=\operatorname{Real}(\lambda)$ is damping ratio, $\lambda_{i}$ is imaginary part $\omega_{m}=\sqrt{\lambda_{i}^{2}}$.

The plate is made of aluminum and the parameters are chosen as follows: the length and width are $a=b=1 \mathrm{~m}$, the thickness $h=10 \mathrm{~mm}$, the thickness of the core layer $h_{c}=9 \mathrm{~mm}$, the thickness of honeycomb wall $t=1.5 \mathrm{~mm}$, the angle $\theta=30^{\circ} . M_{\infty}=3, a_{\infty}=213.36 \mathrm{~m} / \mathrm{s}$. Figure 5 shows the change of the real part $\lambda_{r}$ and frequencies with the flow pressure $P_{\infty}$, when $M=2,4,6$, respectively.

It can be seen from Figure 5 that with the flow pressure increase, the first-order frequency of the system gradually increases and the second-order frequency decreases, and the two gradually get closer and closer. Simultaneously, the vibrations of the first two modes of the zero Poisson's ratio honeycomb sandwich plate are coupled with each other and the system gradually absorbs energy from the airflow. When the two frequencies are the same and at the same time the real part of the eigenvalue curve crosses the zero-line, flutter occurs. The corresponding flow pressure is called critical flow pressure. While due to the existence of structural and aerodynamic damping, the critical flow pressure from the real part curve by the left column of Figure 5 is slightly larger than the value when the corresponding frequencies are combined by the right column of Figure 5 . It can be seen from Figure $5 \mathrm{a}-\mathrm{c}$ that the critical flow pressures are $989 \mathrm{kPa}, 1341 \mathrm{kPa}$ and $1341 \mathrm{kPa}$ when $M=2,4$ and 6 respectively. That is to say, the results are almost same when $M=4$ and 6 , Therefore, take $M=6$ to ensure the correctness of the results. 

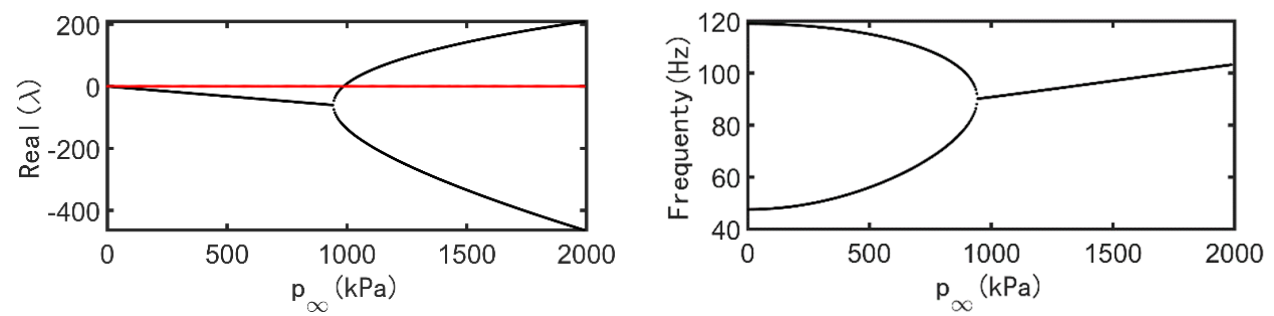

(a)
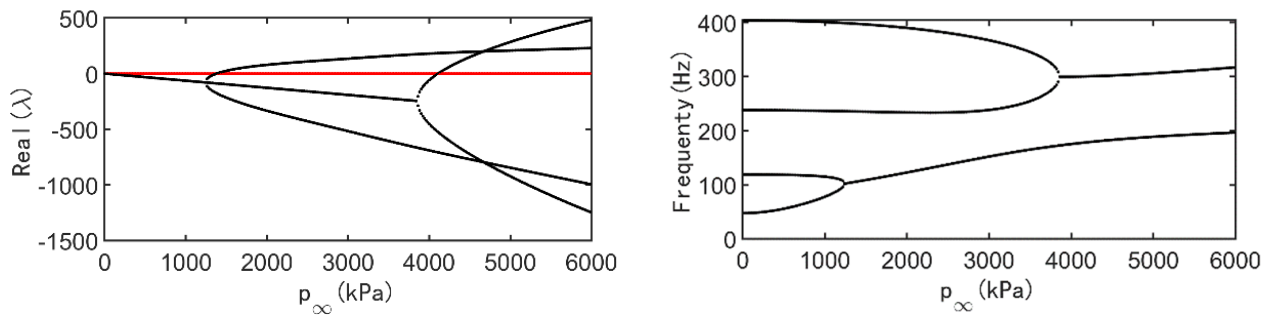

(b)
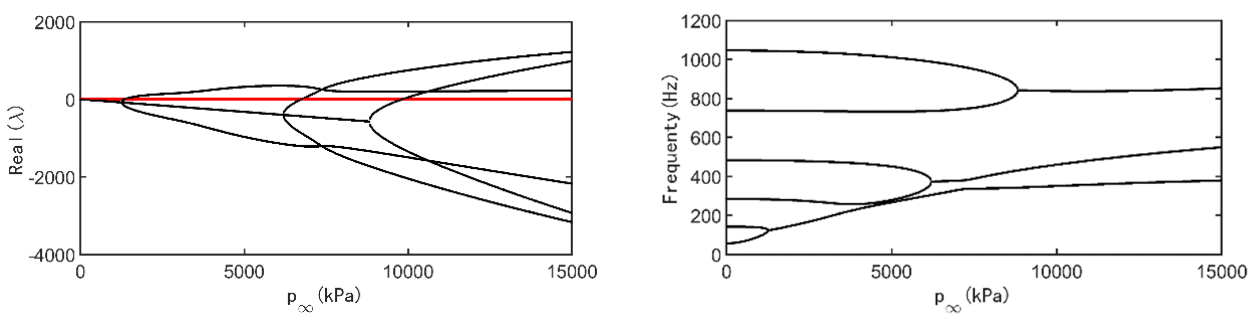

(c)

Figure 5. The curves of eigenvalue with the flow pressure change. (a) $M=2$, (b) $M=4$, (c) $M=6$.

Figure 6 gives the waveform and phase diagrams of the plate under different airflows. Figure $6 \mathrm{a}$ is the stable motions of the ZPR honeycomb sandwich plate when the airflow is less than the critical flow pressure. When the airflow is equal to critical flow pressure in Figure $6 b$, the plate shows periodic motions, which is called limit cycle. Figure 6c expresses divergent motions when the airflow is larger than the critical flow pressure. The phase diagram is an outward spiral as shown in the right side of Figure 6c.

Change the parameters of the zero Poisson's ratio honeycomb sandwich plate and study the influence of these parameters on the flutter characteristics of the plate. Table 8 shows the critical dynamic flow pressure and the corresponding core thickness ratio. The thickness of the plate $h=10 \mathrm{~mm}, t=1 \mathrm{~mm}$, other parameters are same as that of Figure 5 . As the core thickness ratio increases, that is, the total thickness of the plate remains unchanged, when the thickness of the core layer increases and the thickness of the skins decreases, the critical dynamic flow pressure for flutter of the plate gradually decreases.

Table 8. Flow pressures with core thickness ration change.

\begin{tabular}{ccccccc}
\hline Core thickness ratios $\boldsymbol{h}_{\boldsymbol{c}} / \boldsymbol{h}$ & 0.7 & 0.75 & 0.8 & 0.85 & 0.9 & 0.95 \\
\hline Flow pressure $\boldsymbol{P}_{\infty}(\mathrm{kpa})$ & 3321 & 2911 & 2441 & 1921 & 1351 & 701 \\
\hline
\end{tabular}

Changing the thickness of honeycomb wall, with the same other parameters as those of Figure 5, the corresponding critical dynamic flow pressures are shown as in Table 9. It can be obtained from Table 9 that as the wall thickness of the honeycomb cell increases, the critical dynamic flow pressure of flutter first decreases and then increases. When the wall thickness of the honeycomb cell is $1.5 \mathrm{~mm}$, the critical dynamic pressure is the smallest. The influence of wall thickness $t$ is smaller than that of core thickness ratio. 

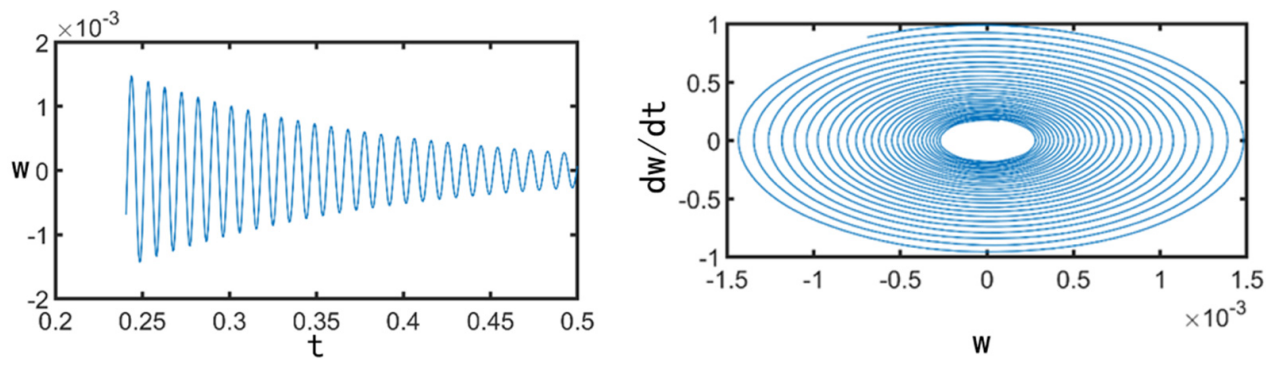

(a)
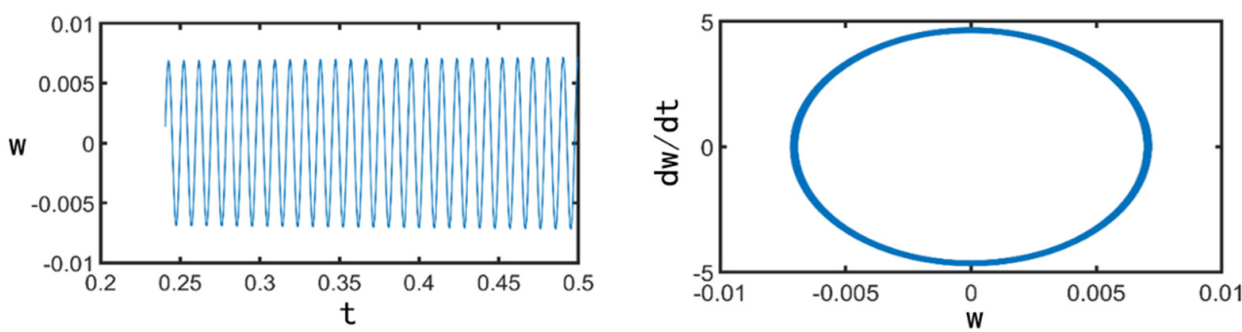

(b)
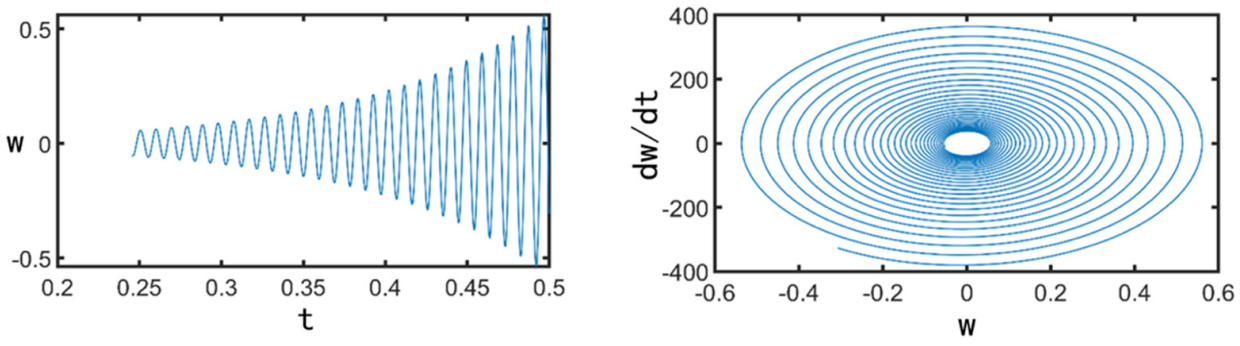

(c)

Figure 6. The waveform and phase diagrams under different flow pressures. (a) $P \infty=1310 \mathrm{kpa}$, (b) $P \infty=1341 \mathrm{kpa},(\mathbf{c}) P \infty=1351 \mathrm{kpa}$.

Table 9. Flow pressures with wall thickness change.

\begin{tabular}{ccccccc}
\hline Wall thickness $t$ & $0.5 \mathrm{~mm}$ & $1 \mathrm{~mm}$ & $1.5 \mathrm{~mm}$ & $2 \mathrm{~mm}$ & $2.5 \mathrm{~mm}$ & $3 \mathrm{~mm}$ \\
\hline Flow pressure $\boldsymbol{P}_{\infty}(\mathrm{kpa})$ & 1381 & 1351 & 1341 & 1351 & 1381 & 1441 \\
\hline
\end{tabular}

With the angle $\theta$ changed and other parameters the same as those of Figure 5, the critical dynamic flow pressures are shown as in Table 10. When the angle increases, the critical dynamic flow pressure decreases slightly.

Table 10. Flow pressures with angle $\theta$ change.

\begin{tabular}{ccccc}
\hline Angle $\theta$ & $15^{\circ}$ & $30^{\circ}$ & $45^{\circ}$ & $60^{\circ}$ \\
\hline Flow pressure $\boldsymbol{P}_{\infty}(\mathrm{kpa})$ & 1381 & 1351 & 1311 & 1281 \\
\hline
\end{tabular}

Changing the aspect ratio of the plate and with the other parameters the same as that of Figure 5, the critical dynamic flow pressures are shown as in Table 11. It can be obtained from Table 11 that as the length-width ratio $b / a$ increases, the critical dynamic flow pressure for flutter decreases. It is concluded that the critical dynamic flow pressure of the square plate is larger than that of the rectangular plate under the boundary condition of four sides simply supported. 
Table 11. Flow pressures with length-width ratio change.

\begin{tabular}{cccccc}
\hline length-width ratio $b / a$ & 1 & 1.5 & 2 & 2.5 & 3 \\
\hline Flow pressure $\boldsymbol{P}_{\infty}(\mathrm{kpa})$ & 1351 & 1061 & 981 & 941 & 921 \\
\hline
\end{tabular}

By comparing the flutter characteristics of the honeycomb sandwich plate with zero Poisson's ratio under different parameters, it can be found that the core thickness ratio has great influence on the critical flutter pressure, and the honeycomb cell wall thickness and cell inclination have a small influence on the critical flutter pressure and the square plate has better stability in supersonic airflow under four sides simply supported boundary.

\section{Conclusions}

The vibrations and flutter of a honeycomb sandwich plate with zero Poisson's ratio are investigated. The equivalent elastic parameters of the hexagonal ZPR honeycomb core are derived and then the dynamical model of the plate is established. The main conclusions are as follows:

1. The validity of the equivalent parameters for the ZPR honeycomb core layer and the sandwich plate model is verified by finite element. The concave hexagonal shape plays a leading role in the hybrid AuxHex honeycomb when force is applied in $y$ direction.

2. The natural frequencies of the sandwich plate are compared when the Poisson's ratios of the core layer are positive, negative and zero, respectively, with the other parameters of the plate remaining the same. The frequencies of the ZPR plate are in the middle and the auxetic plates are the smallest.

3. The change of the natural frequencies of the sandwich plate with the variety of core thickness ratio and length-width ratio of the plate are obtained, with the variety of the honeycomb cells including the length of walls, the angle of walls and the thickness of walls, respectively.

4. The critical dynamic pressure of flutter of the honeycomb sandwich plate is calculated. The critical dynamic pressures decrease when the core thickness ratio and lengthwidth ratio increase, respectively. The critical dynamic pressure decreases first and then increases when the thickness of wall increases.

Author Contributions: Conceptualization, J.Z.; methodology, J.Z. and Z.Y.; software, Z.Y.; validation, J.Z., Z.Y. and L.X.; formal analysis, J.Z. and L.X.; investigation, J.Z. and Z.Y.; resources, J.Z. and L.X.; data curation, Z.Y. and L.X.; writing-original draft preparation, J.Z. and Z.Y.; writing-review and editing, J.Z. and L.X.; supervision, J.Z.; funding acquisition, L.X. and J.Z. All authors have read and agreed to the published version of the manuscript.

Funding: The authors gratefully acknowledge the support of National Natural Science Foundation of China No. 12072041, No. 11732005, Hebei Provincial Natural Science Foundation of China No. A2019202342.

Institutional Review Board Statement: Not applicable.

Informed Consent Statement: Not applicable.

Data Availability Statement: Not applicable.

Conflicts of Interest: The authors declare no conflict of interest.

\section{References}

1. Yan, L.L.; Zhu, K.Y.; Chen, N.; Zheng, X.T.; Quaresimin, M. Energy-absorption characteristics of tube-reinforced absorbent honeycomb sandwich structure. Compos. Struct. 2021, 255, 112946. [CrossRef]

2. Rapaka, S.D.; Pandey, M.; Annabattula, R.K. Dynamic compressive behaviour of auxetic and non-auxetic hexagonal honeycombs with entrapped gas. Int. J. Impact Eng. 2020, 146, 103718. [CrossRef]

3. Rahman, O.; Koohbor, B. Optimization of energy absorption performance of polymer honeycombs by density gradation. Composites Part C: Open Access 2020, 3, 100052. [CrossRef] 
4. Zhang, X.C.; An, C.C.; Shen, Z.F.; Wu, H.X.; Yang, W.G.; Bai, J.P. Dynamic crushing responses of bio-inspired re-entrant auxetic honeycombs under in-plane impact loading. Mater. Today Commun. 2020, 23, 100918. [CrossRef]

5. Zhang, X.C.; An, L.Q.; Ding, H.M.; Zhu, X.Y.; El-Rich, M. The influence of cell micro-structure on the in-plane dynamic crushing of honeycombs with negative Poisson's ratio. J. Sandw. Struct. Mater. 2015, 17, 26-55. [CrossRef]

6. Jin, Y.; Shi, Y.G.; Yu, C.; Wei, G.T.; Hua, B.; Wu, L.Z. A multifunctional honeycomb meta-structure for vibration suppression. Int. J. Mech. Sci. 2020, 188, 105964. [CrossRef]

7. Olympio, K.R.; Gandhi, F. Flexible Skins for Morphing Aircraft Using Cellular Honeycomb Cores. J. Intell. Mater. Syst. Struct. 2010, 21, 1719-1735. [CrossRef]

8. Chen, J.J.; Shen, X.; Li, J.F. Zero Poisson's ratio flexible skin for potential two-dimensional wing morphing. Aerosp. Sci. Technol. 2015, 45, 228-241. [CrossRef]

9. Bubert, E.B.; Woods, K.S.; Lee, K.; Kothera, C.S. Design and fabrication of a passive 1D morphing aircraft skin. J. Intell. Mater. Syst. Struct. 2010, 21, 1699-1717. [CrossRef]

10. Zou, T.T.; Zhou, L. Mechanical property analysis and experimental demonstration of zero Poisson's ratio mixed cruciform honeycomb. Mater. Res. Express 2017, 4, 045702. [CrossRef]

11. Liu, W.D.; Li, H.L.; Zhang, J.; Li, H.D. Elastic properties of a novel cellular structure with trapezoidal beams. Aerosp. Sci. Technol. 2018, 75, 315-328. [CrossRef]

12. Huang, J.; Gong, X.B.; Zhang, Q.H.; Scarpa, F.; Liu, Y.J.; Leng, J.S. In-plane mechanics of a novel zero poisson's ratio honeycomb core. Compos. Part B 2016, 89, 67-76. [CrossRef]

13. Huang, J.; Zhang, Q.H.; Scarpab, F.; Liu, Y.J.; Leng, J.S. Multi-stiffness topology optimization of zero poisson's ratio cellular structures. Compos. Part B 2018, 140, 35-43. [CrossRef]

14. Xu, M.C.; Xu, Z.R.; Zhang, Z.; Lei, H.S.; Bai, Y.C.; Fang, D.N. Mechanical properties and energy absorption capability of AuxHex structure under in-plane compression: Theoretical and experimental studies. Int. J. Mech. Sci. 2019, 159, 43-57. [CrossRef]

15. Xu, M.C.; Liu, D.B.; Wang, P.D.; Zhang, Z.; Jia, H.R.; Lei, H.S.; Fang, D.N. In-plane compression behavior of hybrid honeycomb metastructures: Theoretical and experimental studies. Aerosp. Sci. Technol. 2020, 106, 106081. [CrossRef]

16. Wu, H.X.; Zhang, X.C.; Liu, Y. In-plane crushing behavior of density graded cross-circular honeycombs with zero Poisson's ratio. Thin-Walled Struct. 2020, 151, 106767. [CrossRef]

17. Wang, Y.J.; Zhang, Z.J.; Xue, X.M.; Zhang, L. Free vibration analysis of composite sandwich panels with hierarchical honeycomb sandwich core. Thin-Walled Struct. 2019, 145, 106425. [CrossRef]

18. Zhang, Z.J.; Han, B.; Zhang, Q.C.; Jin, F. Free vibration analysis of sandwich beams with honeycomb-corrugation hybrid cores. Compos. Struct. 2017, 171, 335-344. [CrossRef]

19. Wang, X.C.; Yang, Z.C.; Chen, Z.L.; Gu, Y.S.; Wang, W.Q.; Zhao, Y.; Wang, Y.M. Study on coupled modes panel flutter stability using an energy method. J. Sound Vib. 2020, 468, 115051. [CrossRef]

20. Guimarães, T.A.M.; Marques, F.D.; Ferreira, A.J.M. On the modeling of nonlinear supersonic flutter of multibay composite panels. Compos. Struct. 2020, 232, 111522. [CrossRef]

21. Song, Z.G.; Chen, Y.Y.; Li, Z.Y.; Sha, J.C.; Li, F.M. Axially functionally graded beams and panels in supersonic airflow and their excellent capability for passive flutter suppression. Aerosp. Sci. Technol. 2019, 92, 668-675. [CrossRef]

22. Mahmoudkhani, S. Aerothermoelastic analysis of imperfect FG cylindrical shells in supersonic flow. Compos. Struct. 2019, 225, 111160. [CrossRef]

23. Dhital, K.; Han, J.H. Panel flutter emulation using a few concentrated forces. Int. J. Aeronaut. Space Sci. 2018, 19, 80-88. [CrossRef]

24. Torabi, K.; Afshari, H.; Aboutalebi, F.H. Vibration and flutter analyses of cantilever trapezoidal honeycomb sandwich plates. J. Sandw. Struct. Mater. 2017, 21, 109963621772874. [CrossRef]

25. Saidi, A.R.; Bahaadini, R.; Majidi-Mozafari, K. On vibration and stability analysis of porous plates reinforced by graphene platelets under aerodynamical loading. Compos. Part B 2019, 164, 778-799. [CrossRef]

26. Zhang, W.; Chen, L.L.; Guo, X.Y.; Sun, L. Nonlinear dynamical behaviors of deploying wings in subsonic air flow. J. Fluids Struct. 2017, 74, 340-355. [CrossRef]

27. Ma, L.; Yao, M.H.; Zhang, W.; Cao, D.X. Bifurcation and dynamic behavior analysis of a rotating cantilever plate in subsonic airflow. Appl. Math. Mech. Engl. Ed. 2020, 41, 1861-1880. [CrossRef]

28. Hao, Y.X.; Niu, Y.; Zhang, W.; Li, S.B.; Yao, M.H.; Wang, A.W. Supersonic flutter analysis of FGM shallow conical panel accounting for thermal effects. Meccanica 2018, 53, 95-109. [CrossRef]

29. Yang, S.W.; Zhang, W.; Hao, Y.X.; Niu, Y. Nonlinear vibrations of FGM truncated conical shell under aerodynamics and in-plane force along meridian near internal resonances. Thin-Walled Struct. 2019, 142, 369-391. [CrossRef]

30. Olympio, K.R.; Gandhi, F. Zero Poisson's ratio cellular honeycombs for flex skins undergoing one-dimensional morphing. J. Intell. Mater. Syst. Struct. 2010, 21, 1737-1753. [CrossRef]

31. Gibson, L.J.; Ashby, M.F. Cellular Solids: Structure and Properties; Cambridge University Press: Cambridge, UK, 1997. 